\title{
Retrospective analysis of open surgical versus laser interstitial thermal therapy callosotomy in pediatric patients with refractory epilepsy
}

\author{
James P. Caruso, MD, ${ }^{1}$ M. Burhan Janjua, MD, ${ }^{1}$ Alison Dolce, MD, ${ }^{2}$ and Angela V. Price, $M D^{1}$ \\ Departments of ${ }^{1}$ Neurological Surgery and ${ }^{2}$ Neurology, Children's Medical Center, University of Texas Southwestern, Dallas, \\ Texas
}

OBJECTIVE Corpus callosotomy remains an established surgical treatment for certain types of medically refractory epilepsy in pediatric patients. While the traditional surgical approach is often well tolerated, the advent of MR-guided laser interstitial thermal therapy (LITT) provides a new opportunity to ablate the callosal body in a minimally invasive fashion and minimize the risks associated with an open interhemispheric approach. However, the literature is sparse regarding the comparative efficacy and safety profiles of open corpus callosotomy (OCC) and LITT callosotomy. To this end, the authors present a novel retrospective analysis comparing the efficacy and safety of these methods.

METHODS Patients who underwent OCC and LITT callosotomy during the period from 2005 to 2018 were included in a single-center retrospective analysis. Patient demographic and procedural variables were collected, including length of stay, procedural blood loss, corticosteroid requirements, postsurgical complications, and postoperative disposition. Preand postoperative seizure frequency (according to seizure type) were recorded.

RESULTS In total, 19 patients, who underwent 24 interventions (16 OCC and 8 LITT), were included in the analysis. The mean follow-up durations for the OCC and LITT cohorts were 83.5 months and 12.3 months, respectively. Both groups experienced reduced frequencies of seizure and drop attack frequency postoperatively. Additionally, LITT callosotomy was associated with a significant decrease in estimated blood loss and decreased length of pediatric ICU stay, with a trend of shorter length of hospitalization.

CONCLUSIONS Longer-term follow-up and a larger population are required to further delineate the comparative efficacies of LITT callosotomy and OCC for the treatment of pediatric medically refractory epilepsy. However, the authors' data demonstrate that LITT shows promise as a safe and effective alternative to OCC.

https://thejns.org/doi/abs/10.3171/2020.7.PEDS20167

KEYWORDS corpus callosotomy; laser interstitial thermal therapy; LITT; medical refractory epilepsy; epilepsy surgery; drop attacks

$\mathrm{M}$ EDICALLY refractory epilepsy remains a debilitating pathology in pediatric patients. Multiple antiepileptic agents are often difficult for pediatric patients to tolerate, and children with epilepsy are at increased risk for neurocognitive and developmental delays, even when patients have well-controlled seizures. ${ }^{1-3}$ Additionally, these deficits create a substantial financial burden for patients and their families. ${ }^{4}$ Therefore, epilepsy surgery is a valuable treatment option when patients' seizures be- come refractory to pharmacological therapies. When successful, surgery can eliminate or reduce patients' seizure burden and decrease their dependence on pharmacological therapy.

However, for patients without individual localizing lesions, multifocal epileptogenesis, or generalized epilepsy syndromes, focal resection of epileptogenic foci is ineffective, and other surgical options warrant consideration. For these patients, corpus callosotomy remains an effective

ABBREVIATIONS DTI = diffusion tensor imaging; $\mathrm{LITT}=$ laser interstitial thermal therapy; OCC = open corpus callosotomy; PICU = pediatric ICU. SUBMITTED March 17, 2020. ACCEPTED July 28, 2020.

INCLUDE WHEN CITING Published online January 22, 2021; DOI: 10.3171/2020.7.PEDS20167. 
and well-tolerated surgical treatment. ${ }^{5}$ Since the corpus callosum provides reciprocal connections for nearly $80 \%$ of the cerebral cortex volume, a callosotomy can reduce seizure burden by decreasing interhemispheric propagation of epileptiform activity. ${ }^{6,7}$ Van Wagenen and Herren first described a surgical technique for corpus callosotomy in 1940 in a study of refractory seizures in patients with high-grade gliomas, ${ }^{8}$ and further studies substantiated the impact of this treatment approach. ${ }^{9,10}$ While relatively few patients experience complete seizure freedom after corpus callosotomy, certain types of seizures are particularly responsive to treatment. Specifically, corpus callosotomy is associated with notable postoperative reductions in tonic and atonic drop attacks as well as partial seizures with rapid secondary generalization. ${ }^{5,11,12}$

Despite its success, corpus callosotomy does pose a risk of undesirable surgical complications, including disconnection syndrome and injury to the pericallosal arteries. As a result, numerous recent studies focus on less invasive alternatives to open surgical corpus callosotomy. Studies have shown that radiosurgical callosal ablation is a noninferior alternative to open callosotomy, but the cognitive risks of radiation limit its application in pediatric populations. ${ }^{13}$ Endoscopic techniques for callosum resection are becoming more commonplace, but the expertise required to perform these procedures restricts the procedure's availability, and limitations of operative field and instrumentation mobility become more burdensome when repairing an unintended vascular injury. ${ }^{14}$

MR-guided laser interstitial thermal therapy (LITT) is another proposed alternative to open corpus callosotomy (OCC) to achieve comparable seizure relief while minimizing surgical complications. Most LITT literature focuses on the outcomes and safety of tumor ablation performed with this technique, and recent advances have facilitated its application to cases that were previously considered nonoperative. ${ }^{15}$ Additionally, data suggest that LITT is an effective treatment for multiple pediatric epileptogenic pathologies, including hypothalamic hamartoma, focal cortical dysplasia, mesial temporal sclerosis, and cortical tubers in patients with tuberous sclerosis. ${ }^{16}$ Some reported studies have clarified the role of LITT in the treatment of nonlesional or generalized epilepsy, especially in comparison to well-established open callosotomy techniques, ${ }^{17-26}$ but more investigation is needed to compare different methods of callosotomy. To this end, the authors present what is to their knowledge the first retrospective analysis including both LITT and open callosotomy patients with medically refractory epilepsy.

\section{Methods \\ Data Collection}

All patients treated at a single center with either surgical OCC or LITT callosotomy from 2005 to 2018 were included in the analysis. The study received IRB approval, and demographic and procedural variables were collected from review of the electronic medical record. All cases were reviewed at the institution's multidisciplinary epilepsy conference. All procedures were performed by a single surgeon (A.V.P.). For each patient, demographic informa- tion included age, sex, medical comorbidities, and epilepsy etiology. Seizure frequency data and seizure rates were calculated from retrospective chart review for the duration of the study, based on neurology and neurosurgery clinic and inpatient notes. The types of seizures recorded included drop attacks, as well as tonic, tonic-clonic, and myoclonic seizures. Seizure outcomes were assessed at the last follow-up. The authors compared the incidence of postprocedure complications, need for revision procedure, length of hospital stay, and postoperative disposition between groups.

\section{Statistical Analysis}

To analyze differences in procedural variables for OCC and LITT cases, paired t-tests were used. The ShapiroWilks test was applied to evaluate whether data were normally distributed. When data were not normally distributed, Wilcoxon signed-rank tests were used. For comparisons of continuous and ordinal variables between the OCC and LITT groups, the Mann-Whitney U-test was applied. Statistical significance was reported with a p value $<0.05$, with a Bonferroni correction applied in cases of multiple comparisons. All statistical analysis was performed using R version 3.3.3 (R Foundation for Statistical Computing, 2017).

\section{OOC Operative Technique}

After administration of general anesthesia, patients are positioned supine with the head mildly flexed and secured using a Mayfield frame. An S-shaped partial bicoronal incision site is marked $1 \mathrm{~cm}$ anterior to the coronal suture. After standard sterile preparation, the incision is opened using micropoint cautery, and the skull is exposed in standard fashion with electrocautery and periosteal elevation. The coronal and sagittal sutures are identified during skull exposure, and three burr holes are planned. Two burr holes are located in the midline, $2 \mathrm{~cm}$ anterior to the coronal suture and $1 \mathrm{~cm}$ posterior to the coronal suture, and the third burr hole is located laterally directly over the coronal suture. Following elevation of the craniotomy, the dura is opened in a C-shaped fashion, with the anchoring limb reflected over the sagittal sinus for protection. Once the parenchyma is exposed, the remaining interhemispheric approach is performed with the surgical microscope. With the assistance of a retraction system to mobilize the frontal lobe, meticulous dissection is performed to expose the corpus callosum, with special care to avoid sacrifice of superficial bridging veins and the pericallosal arteries. The corpus callosum lesioning is performed with gentle suction, bipolar cautery, and blunt dissection until the ependyma of the lateral ventricle is exposed. If the lateral wall of the ventricle is breached, an external ventricular drain is placed for postoperative CSF diversion. Postoperatively, patients are monitored in the ICU for hemodynamic and neurological stability.

\section{LITT Preoperative Planning}

At the institution's multidisciplinary epilepsy conference, patients were considered for corpus callosotomy based on the patients' clinical symptoms, seizure burden, 


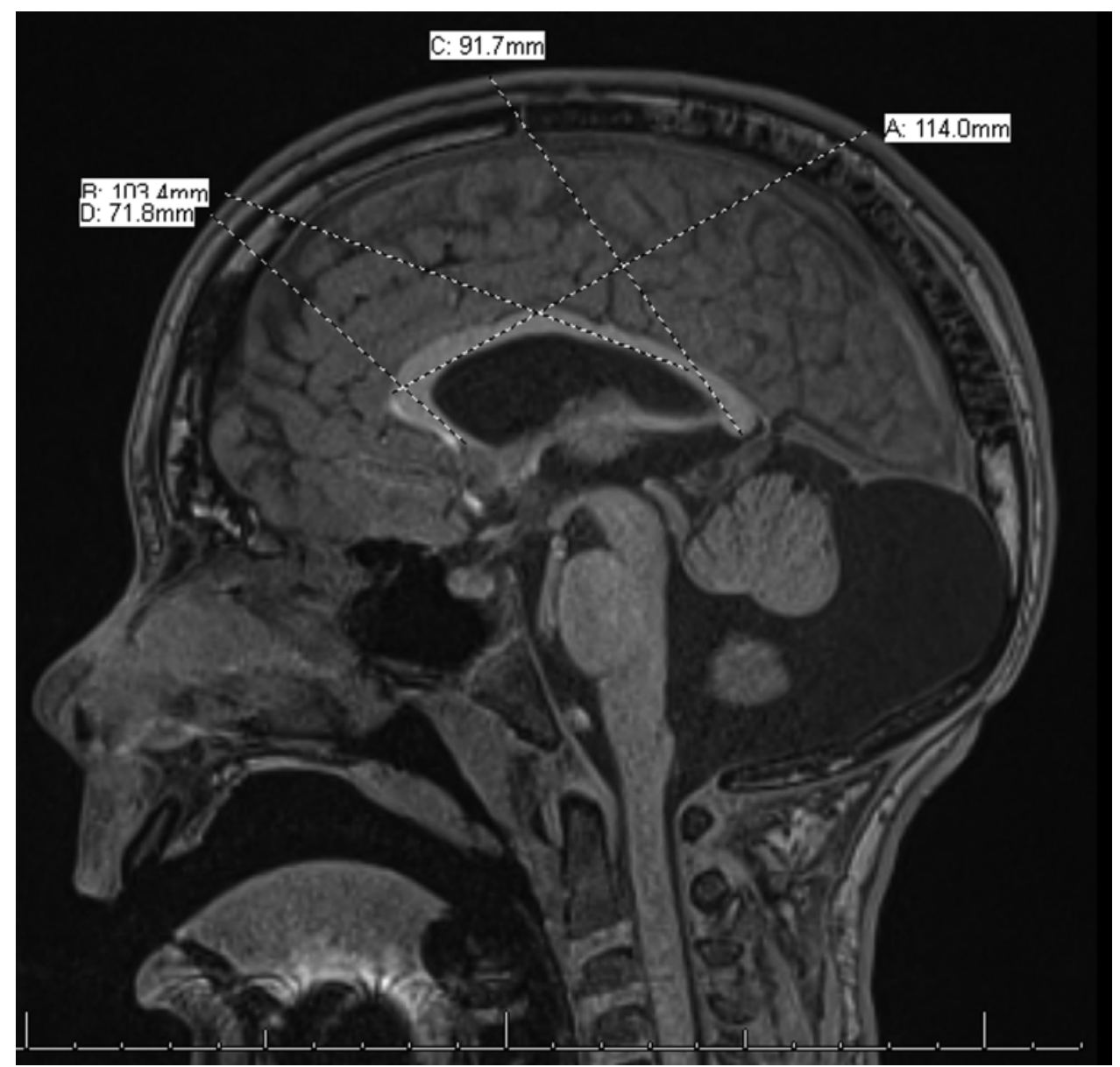

FIG. 1. Sagittal MRI of the brain of a patient, establishing preoperative planning of four trajectories for an LITT complete callosotomy.

imaging findings, and efficacy of current antiepileptic medication regimen. Prior to June 2017, OCC was performed in all cases because LITT was unavailable. Once LITT became available at the institution, the senior surgeon (A.V.P.) offered both LITT and OCC to patients who qualified for callosotomy. Exclusion criteria for LITT callosotomy included patients who could not undergo MRI and patients with intracranial pathology that precluded safe placement and targeting of the fibers. If patients and their families agreed to LITT callosotomy, preoperative MR images ( $\mathrm{T} 1$ axial postcontrast and $\mathrm{T} 2$ axial/coronal/ sagittal sequences) were analyzed for the planning of between two and four trajectories using image-guidance software. The number of trajectories used and the decision to pursue an anterior two-thirds callosotomy or complete callosotomy were determined based on the patient's clinical history and the preference of the primary surgeon. The following provides a methodological outline of a standard trajectory plan: 1) the rostrum of the corpus callosum was targeted with an anterior trajectory parallel to the rostrum in the sagittal plane, 2) the genu and anterior one-third of the body were targeted with a single trajectory originating behind the coronal suture, 3 ) the posterior two-thirds of the body was targeted with a single horizontal trajec- tory with the entry point on the superior forehead when possible, and 4) the splenium was targeted with a parallel fiber trajectory originating in the parietal region. Importantly, trajectories were planned to avoid crossing sulci or large vessels, with particular care to avoid the plane of the pericallosal arteries and lateral ventricle margin. Eloquent cortex was avoided whenever possible. Bilateral trajectories were used to reduce the risk of thermal injury and avoid crossing of individual trajectory paths. Figure 1 demonstrates trajectory planning for an example case. $\mathrm{Pa}$ tients were treated with oral corticosteroids the day prior to LITT, and preoperative trajectories were finalized using the registration MRI during the procedure.

\section{LITT Intraoperative Methodology}

Patients were positioned supine with the head mildly flexed or in a neutral position anchored in a stereotactic Cosman-Roberts-Wells (CRW) frame. All patients were treated with the Visualase system (Medtronic). A registration MRI was performed, which was merged with the preoperative MRI containing the preplanned trajectories. Planned trajectories extend from the skin surface to the target area, and the stereotactic frame is used to identify 


\section{Pt 1}

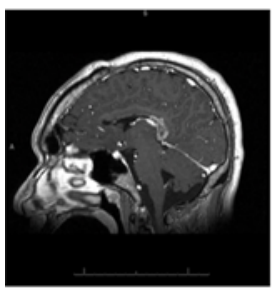

Pt 2

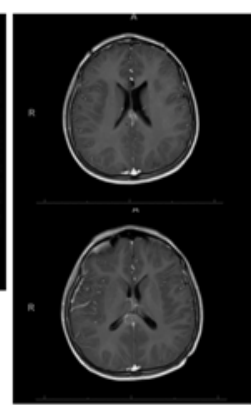

Pt 3

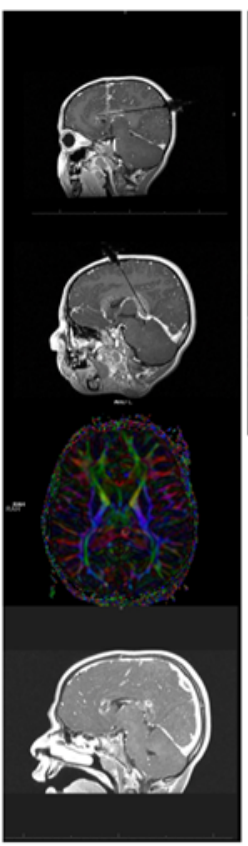

Pt 4

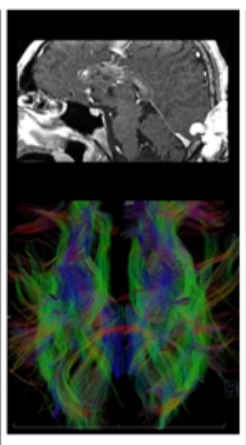

Pt 5

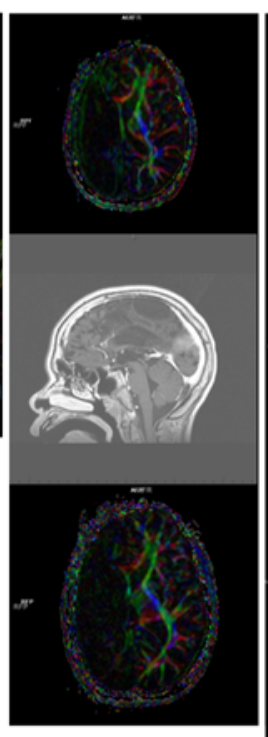

Pt 6

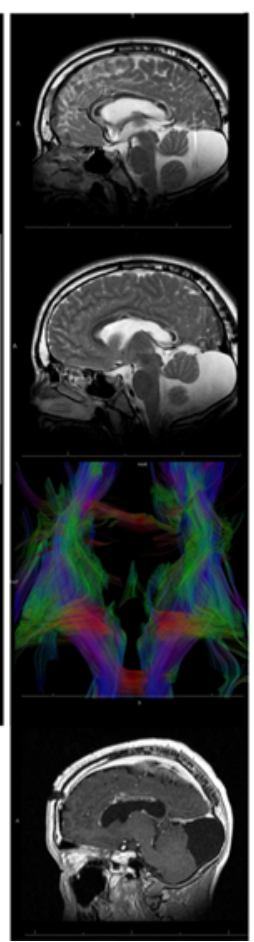

Pt 7

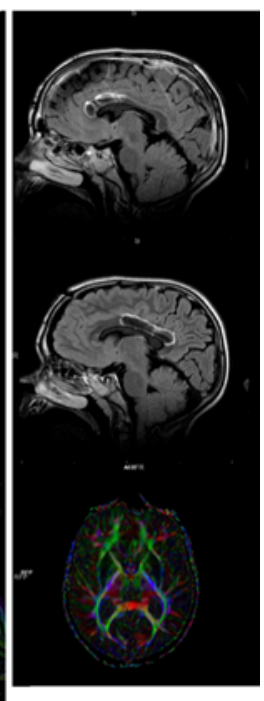

FIG. 2. Pre- and postoperative imaging for select LITT patients. Pt 1: Immediate postoperative results after LITT posterior corpus callosotomy. Pt 2: Immediate postoperative results after LITT (body) (upper) and immediate postoperative results after LITT (splenium) (lower). Pt 3 (top to bottom): Immediate postoperative results after LITT (body) and after LITT (splenium); DTI 8 months after LITT demonstrating residual genu and splenium connections; and immediate postoperative results after second LITT of residual connections. Pt 4: Immediate postoperative results after LITT complete corpus callosotomy (upper) and DTI 12 months after LITT demonstrating residual splenium connections (lower). Pt 5: DTI after hemispherotomy (precallosotomy) showing residual genu connections (upper); immediate postoperative results after LITT demonstrating residual genu connections (middle); and DTI 14 months after LITT showing decreased residual genu connections (lower). Pt 6 (top to bottom): immediate postoperative results after LITT (genu and splenium) and after LITT (body and splenium); DTI 10 months after LITT demonstrating residual body connections; and immediate postoperative results after second LITT of residual connections. Pt 7: Immediate postoperative results after LITT (genu and body) (upper) and after LITT (body) (middle); and DTI 1 week after LITT showing residual splenium connections (lower). $\mathrm{Pt}=$ patient. Figure is available in color online only.

the trajectories. Small stab incisions and subsequent burr holes are made at the entry sites for each trajectory. After coagulating the dura, the guiding bolts are inserted over the guiding probe. The probes are removed, and the laser fibers are inserted to a predetermined target distance before being secured into the guiding bolt.

Following the insertion of the guiding bolts, the patient was transferred to the intraoperative MRI suite and a localizing brain MRI was performed to ensure accurate placement of the laser fibers. The laser thermal lesioning was then performed under MR-guided thermography to monitor changes in temperature. To achieve ablation of the desired region in the corpus callosum, fibers are gently withdrawn approximately $7-8 \mathrm{~mm}$. When the entire lesioning was complete, another intraoperative MRI was performed to visualize the location of the lesions. If the lesion extent and location were desirable, a contrasted MRI sequence was obtained to document the extent of the ablation. Each laser fiber and bolt were subsequently removed and the incisions closed with absorbable suture.

At completion of the procedure, the patient was trans- ferred to the ICU for neurological and hemodynamic monitoring. High-dose corticosteroids were administered postoperatively to reduce edema in the immediate postoperative period, followed by an extended taper. A follow-up MRI, which revealed a complete corpus callosotomy, was performed for 1 patient after concerns regarding his neurological status 3 months following LITT. Figure 2 shows examples of pre- and postoperative imaging for representative LITT cases.

\section{Results}

The study included 19 patients who underwent OCC or LITT callosotomy for medically refractory epilepsy, which included frequent drop attacks and tonic, tonicclonic, and myoclonic seizures. In total, 24 operations were performed (16 OCC and 8 LITT callosotomies), the male-to-female ratio was $3: 1$, and the mean age in the OCC group was 10.9 years compared with 10.6 years in the LITT group. Of the 14 patients who initially underwent OCC, 4 underwent subsequent procedures ( 2 under- 


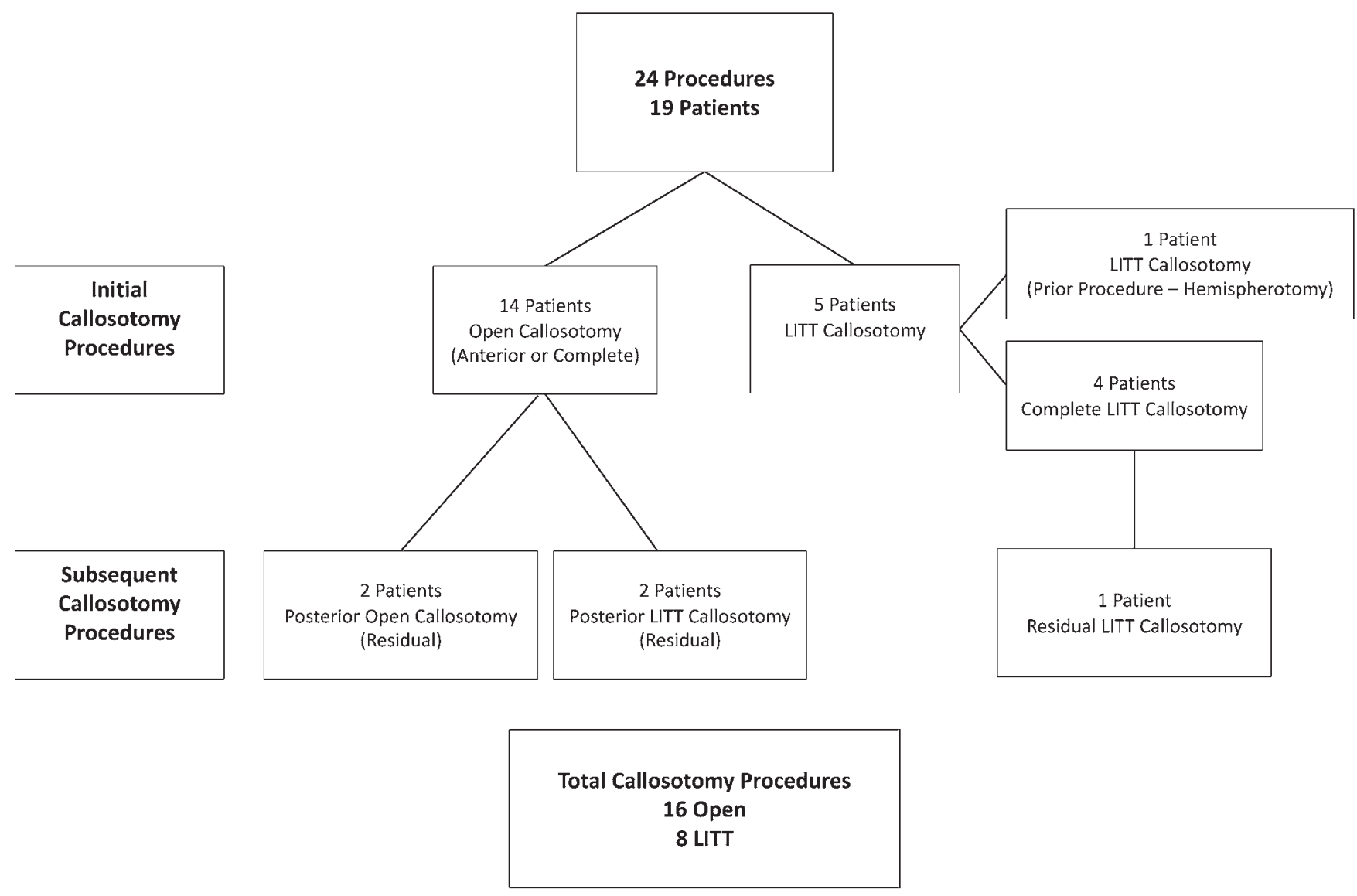

FIG. 3. Breakdown of all patients and the procedures completed.

went OCC for posterior completion of a previous anterior two-thirds callosotomy, and 2 underwent LITT for posterior completion of a previous anterior two-thirds callosotomy). Of the 5 patients who underwent LITT initially, 4 underwent complete LITT callosotomy, and 1 patient underwent LITT for residual connections that were identified following a functional hemispherotomy. One of these 4 initial LITT callosotomy patients underwent subsequent LITT for residual unlesioned connections. Figure 3 illustrates the patient population according to procedures performed, and demographic and clinical characteristics for the included patients are shown in Table 1.

LITT procedures had significantly less mean blood loss compared with OCC procedures (7 vs $84 \mathrm{ml}, \mathrm{p}<0.0001$ ) and a significantly shorter pediatric ICU (PICU) stay (1 vs 2.3 days, $\mathrm{p}<0.001$ ). Additionally, LITT patients demonstrated a trend toward decreased length of stay (4.6 vs 5.8 days, $\mathrm{p}=0.32$ ). Of note, 1 LITT patient had a prolonged length of stay attributable to postoperative venous thrombus formation, diagnosis and workup of newly discovered factor V Leiden deficiency, and CSF leak at one of the incision sites (which resolved with an additional suture). This patient's length of stay was not included in the above analysis. Additionally, operating time was significantly longer in LITT procedures (492 vs 249 minutes, $\mathrm{p}<0.001$ ), and LITT cases with longer operative times also tended to have more planned trajectories and larger volumes of planned callosal lesioning. LITT procedures also required significantly longer durations of postoperative steroid use (13.7 vs 4.4 days, $p<0.001$ ). The observed statistically significant differences above remained after application of Bonferroni correction for multiple comparisons between the OCC and LITT groups. Additionally, while $100 \%$ of LITT patients were discharged home postoperatively, 3 (19\%) OCC patients required a postoperative inpatient rehabilitation course prior to discharge. Table 2 summarizes the hospitalization variables for both groups.

The mean follow-up period after an OCC procedure was 83.5 months (range 20-125 months), compared with 12.3 months (range 6-18 months) after an LITT procedure. Patients scheduled to undergo OCC suffered from a mean of 6.1 drop attacks and 6.7 seizures per day preprocedure. Postprocedure, these values were reduced to 0.33 drop attacks and 1.2 other seizures per day, respectively. Similarly, patients scheduled to undergo LITT suffered from a mean of 4.4 seizures and 8.1 drop attacks per day, and postoperatively these rates were reduced to 1.34 seizures and 0.38 drop attacks per day. Of note, 5 OCC patients underwent additional surgical procedures for epilepsy control. Two patients underwent vagal nerve stimulator placement, 2 patients underwent further resection of individual seizure foci, and 1 patient underwent functional hemispherotomy. While 2 LITT patients underwent additional LITT for residual unlesioned territory, at the time of 
TABLE 1. Patient demographic and clinical characteristics

\begin{tabular}{|c|c|c|}
\hline Variable & $\operatorname{OCC}(n=16)$ & $\operatorname{LITT}(n=8)$ \\
\hline Mean age in yrs & 10.9 & 10.6 \\
\hline \multicolumn{3}{|l|}{ Sex } \\
\hline Male & 10 & 8 \\
\hline Female & 6 & 0 \\
\hline \multicolumn{3}{|l|}{ Epilepsy etiology } \\
\hline Nonaccidental trauma & 1 & 0 \\
\hline Cortical dysplasia & 3 & $\begin{array}{l}2 \text { (same } \\
\text { patient) }\end{array}$ \\
\hline Lennox-Gastaut syndrome & 1 & 2 \\
\hline Tuberous sclerosis & 2 & 0 \\
\hline Congenital CMV/polymicrogyria & 1 & 0 \\
\hline Doose syndrome & 2 & 2 \\
\hline Neonatal stroke & 0 & 1 \\
\hline Cryptogenic & 6 & 1 \\
\hline Duration of intractable epilepsy in yrs & 7.6 & 9.1 \\
\hline Average no. of preop ASMs & 3.13 & 2.88 \\
\hline \multicolumn{3}{|l|}{ Prior neurosurgeries } \\
\hline VNS & 2 & 2 \\
\hline VP shunt & 1 & 0 \\
\hline SEGA resection & 2 & 0 \\
\hline Anterior 2/3 CC (open) & 2 & 2 \\
\hline Functional hemispherotomy & 0 & 1 \\
\hline LITT & 0 & 1 \\
\hline Follow-up in mos (range) & $83.5(20-125)$ & $12.3(6-18)$ \\
\hline
\end{tabular}

$\mathrm{ASM}=$ antiseizure medication $; \mathrm{CC}=$ corpus callosotomy $; \mathrm{CMV}=$ cytomega lovirus; SEGA = subependymal giant cell astrocytoma; VNS = vagus nerve stimulator; VP = ventriculoperitoneal.

Values are presented as the numbers of cases unless otherwise indicated. Assessment of seizure frequency was not available for all subjects.

this report no LITT patients had required additional surgical procedures. Three patients experienced postoperative wound infections ( 2 patients from the OCC group and 1 patient from the LITT group).

\section{Discussion}

Since Van Wagenen's pioneering 1940 analysis, corpus callosotomy has evolved into a well-established palliative surgery for patients with medically refractory epilepsy. ${ }^{5,11,12}$ While callosotomy often does not completely eliminate seizures, it reduces the burden of frequent drop attacks and helps prevent the sequelae of rapidly generalizing partial seizures. ${ }^{5,11,12}$ However, these benefits come with equally well-established risks, including the risk of retraction injury to the cingulate gyrus, vascular injury to the pericallosal arteries, CSF leak from a breach of the lateral ventricle wall, disconnection syndrome, and the standard perioperative risks associated with invasive cranial neurosurgery procedures (including blood loss, prolonged ICU stay and hospitalization, and need for physical rehabilitation following immediate postoperative care). To this end, physicians, patients, and patients' families are be-
TABLE 2. Hospitalization variables

\begin{tabular}{lccc}
\hline & OCC & LITT & p Value \\
\hline Blood loss $(\mathrm{ml})$ & 84 & 7 & $<0.0001$ \\
\hline PICU length of stay (days) & 2.3 & 1 & $<0.001$ \\
\hline Overall length of stay (days) & 5.8 & 4.6 & 0.32 \\
\hline Op duration (mins) & 249 & 492 & $<0.001$ \\
\hline Postop steroid use duration (days) & 4.4 & 13.7 & $<0.001$ \\
\hline
\end{tabular}

Values are presented as means unless otherwise indicated.

coming increasingly interested in less invasive alternatives for surgical treatment of intractable epilepsy, and LITT is becoming a well-established treatment option for patients with other complex neurosurgical pathologies, including surgically inaccessible tumors and radiation necrosis. ${ }^{1,27-30}$

At this time, there is some literature dedicated to the investigation of LITT for treating medically refractory epilepsy, but these analyses are limited to case reports and smaller case series. ${ }^{17-26}$ Additionally, some studies discuss the application of other minimally invasive techniques. Eder et al. reported a reduction in partial seizures with secondary generalization in 3 patients who underwent radiosurgical callosotomy after failing prior surgical epilepsy treatments (functional hemispherotomy and anterior temporal lobectomy), ${ }^{13}$ and Sood et al. reported 4 patients who underwent endoscopic corpus callosotomy successfully. ${ }^{14} \mathrm{Ho}$ et al. have provided a comprehensive technical outline for LITT callosotomy targeted to the splenium, ${ }^{31}$ and Singh et al. have elaborated on the operative technique, with specific anatomical landmarks, for complete and anterior corpus callosotomies..$^{32}$ These cases are both novel and informative, and the authors supplement these contributions with both LITT and OCC cases that facilitate a more direct comparison of the safety and efficacy of these procedures.

As LITT is applied to a variety of neurosurgical pathologies, it is important to establish that it is safe. Among the LITT and OCC groups in the present study, no mortalities or severe neurological deficits were encountered, and no patients demonstrated postoperative evidence of acute or chronic disconnection syndrome or supplementary motor area (SMA) syndrome. The complications encountered in the LITT group all occurred in a single patient who had preoperative factor $\mathrm{V}$ Leiden deficiency uncovered during his hospitalization for the procedure. These complications included wound infection, CSF leak, and postoperative peripheral vein thrombosis, all of which were managed medically and did not result in long-term neurological or functional declines. LITT was also associated with a significantly reduced perioperative blood loss, a significantly decreased length of PICU stay, and a trend toward decreased overall length of stay. The data suggest that LITT is well tolerated in pediatric patients, and further measures can improve patient satisfaction. Obtaining immediate postoperative imaging can identify complications postablation. If the imaging is unremarkable, patients may not require postoperative monitoring in the ICU unless additional medical issues arise. While the patient population in the present study is not large enough and does not possess 


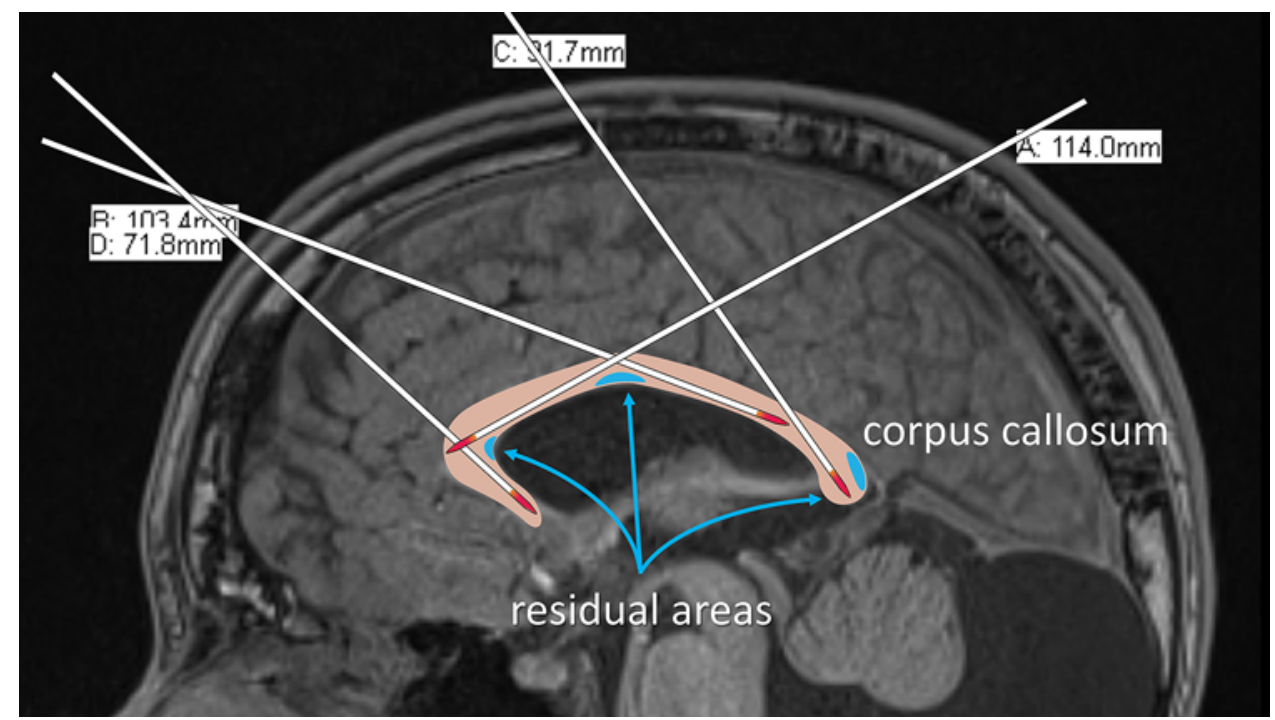

FIG. 4. Potential areas at risk for residual connections following LITT callosotomy. Red-tipped points represent the site of thermocoagulation at the end of the laser fiber. Figure is available in color online only.

sufficient follow-up to provide formal and specific indications for LITT callosotomy versus open callosotomy, the authors' data suggest that LITT is a safe alternative for patients that qualify for callosotomy and do not meet specific exclusion criteria (implants or pathology that precludes fiber placement, inability to undergo or tolerate MRI).

\section{Proposed Improvements in LITT Technique}

Karsy et al. provide a detailed outline of the authors' preferred surgical technique for the LITT procedure. ${ }^{33}$ Preoperative planning is imperative to determine the necessary size of each laser fiber used and the trajectory best suited for lesioning the corpus callosum without damaging eloquent cortex, white matter tracts, or critical vascular structures along the parenchymal surface and adjacent to the corpus callosum. Initial cases in the LITT group were planned as complete callosal ablations, but postoperative diffusion tensor imaging (DTI) revealed small areas of residual unlesioned fibers adjacent to the junctions of different fiber tracts (as shown in Fig. 4). However, later cases utilized between two and four fibers and focused on the anterior two-thirds of the corpus callosum to avoid leaving small residual areas, with plans for a second LITT procedure should seizures continue. It is also possible that attempting complete ablations with a four-fiber plan contributed to a greater degree of postoperative edema, although we did not confirm this with routine postoperative imaging. However, a greater degree of edema would explain the increased need for postoperative steroids and longer hospitalizations in LITT patients. Both the need for postoperative steroids and length of hospitalizations showed improvement after transitioning to an anterior two-thirds LITT callosotomy with fewer fibers.

Additionally, the authors propose that implantation of the laser fiber targeting the callosal body should occur before the implantation of other fibers. This will reduce the possibility of placement error associated with brain shift when placing the extended fiber along the long axis of the callosal body. Moreover, since the corpus callosum is often thinner directly along the midline plane compared with laterally, planning the trajectory of the callosal body fiber to align slightly off midline can better the likelihood of appropriate ablation. Care must be taken when using this modification to prevent injury to the adjacent cingulate gyrus.

\section{Study Limitations and Future Directions}

The authors' analysis shows some limitations. It is a retrospective single-center analysis, and since callosotomies (via open surgical and LITT approaches) are comparatively uncommon procedures, the study included a relatively small number of patients. Additionally, comparisons between OCC patients and LITT patients are limited, since many patients underwent multiple procedures, and the follow-up interval for LITT patients was notably shorter than that of the OCC patients. The heterogeneity of the patient population, limited follow-up interval for patients who underwent LITT, and small sample size preclude a direct comparative analysis of LITT versus OCC for seizure control and reduction. These limitations can be rectified with a larger cohort of patients and a longer duration of follow-up. Additionally, Engel scores were not compared between groups, since complete seizure freedom was not always the goal of intervention, and the follow-up duration varied between the LITT and OCC groups.

With the acquisition of more patient data, and eventual involvement of additional centers and the collection of prospective data, more questions regarding the application of LITT for treating medically refractory epilepsy can be answered. These topics include the demographic and clinical factors predisposing patients to ideal outcomes and the long-term neuropsychological development of patients treated with LITT. Neuropsychological outcomes were not assessed because relatively few patients underwent formal 
pre- and postoperative neuropsychological assessments. However, this is now standard practice at the authors' institution, and comparison of neuropsychological outcomes between OCC and LITT patients remains a viable topic for future study. Additionally, a detailed cost-effectiveness analysis comparing LITT and OCC would determine whether the increased costs associated with prolonged intraoperative time in LITT cases are offset by the reduction in cost associated with reduced ICU duration and the trend toward reduced hospitalization. The authors also observed that patients and their families were more likely to agree to a minimally invasive procedure with a shorter hospital stay, as opposed to an open procedure. However, these observations were anecdotal. Further study of factors influencing patients' willingness to consent to epilepsy surgery would clarify whether a minimally invasive option, such as LITT callosotomy, is more appealing than an open procedure.

\section{Conclusions}

The authors provide the largest available retrospective analysis including both LITT and open callosotomy in pediatric patients with medically refractory epilepsy. LITT is associated with significant reductions in postoperative seizure frequency and drop attack frequency, in addition to reduced intraoperative blood loss and shortened postoperative ICU stay, with a trend toward a decreased length of hospitalization when compared with OCC. LITT demonstrates great promise as an alternative method of performing corpus callosotomy in carefully selected cases, but additional patient data and longer-term follow-up are paramount to confirm LITT durability.

\section{References}

1. Beaumont TL, Mohammadi AM, Kim AH, et al. Magnetic resonance imaging-guided laser interstitial thermal therapy for glioblastoma of the corpus callosum. Neurosurgery. 2018; 83(3):556-565.

2. Bjørnaes H, Stabell K, Henriksen O, Løyning Y. The effects of refractory epilepsy on intellectual functioning in children and adults. A longitudinal study. Seizure. 2001;10(4):250259.

3. Shinnar S, Pellock JM. Update on the epidemiology and prognosis of pediatric epilepsy. J Child Neurol. 2002;17(suppl 1):S4-S17.

4. O'Dell C, Wheless JW, Cloyd J. The personal and financial impact of repetitive or prolonged seizures on the patient and family. J Child Neurol. 2007;22(5 suppl):61S-70S.

5. Graham D, Tisdall MM, Gill D. Corpus callosotomy outcomes in pediatric patients: a systematic review. Epilepsia. 2016;57(7):1053-1068.

6. Kasasbeh AS, Smyth MD, Steger-May K, et al. Outcomes after anterior or complete corpus callosotomy in children. Neurosurgery. 2014;74(1):17-28.

7. Téllez-Zenteno JF, Hernández Ronquillo L, Moien-Afshari F, Wiebe S. Surgical outcomes in lesional and non-lesional epilepsy: a systematic review and meta-analysis. Epilepsy Res. 2010;89(2-3):310-318.

8. Van Wagenen WP, Herren RY. Surgical division of commissural pathways in the corpus callosum: relation to spread of an epileptic attack. Arch Neurol Psychiatry. 1940;44(4): $740-759$.

9. Andersen B, Rogvi-Hansen B, Kruse-Larsen C, Dam M.
Corpus callosotomy: seizure and psychosocial outcome. A 39-month follow-up of 20 patients. Epilepsy Res. 1996;23(1): $77-85$.

10. Kawai K, Shimizu H, Yagishita A, et al. Clinical outcomes after corpus callosotomy in patients with bihemispheric malformations of cortical development. J Neurosurg. 2004;101(1 suppl):7-15.

11. Cendes F, Ragazzo PC, da Costa V, Martins LF. Corpus callosotomy in treatment of medically resistant epilepsy: preliminary results in a pediatric population. Epilepsia. 1993; 34(5):910-917.

12. Wong TT, Kwan SY, Chang KP, et al. Corpus callosotomy in children. Childs Nerv Syst. 2006;22(8):999-1011.

13. Eder HG, Feichtinger M, Pieper T, et al. Gamma knife radiosurgery for callosotomy in children with drug-resistant epilepsy. Childs Nerv Syst. 2006;22(8):1012-1017.

14. Sood S, Marupudi NI, Asano E, et al. Endoscopic corpus callosotomy and hemispherotomy. J Neurosurg Pediatr. 2015; 16(6):681-686.

15. Wilfong AA, Curry DJ. Hypothalamic hamartomas: optimal approach to clinical evaluation and diagnosis. Epilepsia. 2013;54(suppl 9):109-114.

16. Gonzalez-Martinez J, Vadera S, Mullin J, et al. Robotassisted stereotactic laser ablation in medically intractable epilepsy: operative technique. Neurosurgery. 2014;10(suppl 2):167-173.

17. Roland JL, Akbari SHA, Salehi A, Smyth MD. Corpus callosotomy performed with laser interstitial thermal therapy. $J$ Neurosurg. 2021;134(1):314-322.

18. Tao JX, Issa NP, Wu S, et al. Interstitial stereotactic laser anterior corpus callosotomy: a report of 2 cases with operative technique and effectiveness. Neurosurgery. 2019;85(3): E569-E574.

19. Palma AE, Wicks RT, Popli G, Couture DE. Corpus callosotomy via laser interstitial thermal therapy: a case series. $J$ Neurosurg Pediatr. 2018;23(3):303-307.

20. Ball T, Sharma M, White AC, Neimat JS. Anterior corpus callosotomy using laser interstitial thermal therapy for refractory epilepsy. Stereotact Funct Neurosurg. 2018;96(6): 406-411.

21. Tao JX, Satzer D, Issa NP, et al. Stereotactic laser anterior corpus callosotomy for Lennox-Gastaut syndrome. Epilepsia. 2020;61(6):1190-1200.

22. Badger CA, Lopez AJ, Heuer G, Kennedy BC. Systematic review of corpus callosotomy utilizing MRI guided laser interstitial thermal therapy. J Clin Neurosci. 2020;76:67-73.

23. Vakharia VN, Sparks RE, Vos SB, et al. Computer-assisted planning for minimally invasive anterior two-thirds laser corpus callosotomy: a feasibility study with probabilistic tractography validation. Neuroimage Clin. 2020;25:102174.

24. Lehner KR, Yeagle EM, Argyelan M, et al. Validation of corpus callosotomy after laser interstitial thermal therapy: a multimodal approach. J Neurosurg. 2019;131(4):1095-1105.

25. Youngerman BE, Save AV, McKhann GM. Magnetic resonance imaging-guided laser interstitial thermal therapy for epilepsy: systematic review of technique, indications, and outcomes. Neurosurgery. 2020;86(4):E366-E382.

26. Huang Y, Yecies D, Bruckert L, et al. Stereotactic laser ablation for completion corpus callosotomy. J Neurosurg Pediatr. 2019;24(4):433-441.

27. Carpentier A, McNichols RJ, Stafford RJ, et al. Laser thermal therapy: real-time MRI-guided and computer-controlled procedures for metastatic brain tumors. Lasers Surg Med. 2011; 43(10):943-950.

28. Diaz R, Ivan ME, Hanft S, et al. Laser interstitial thermal therapy: lighting the way to a new treatment option in neurosurgery. Neurosurgery. 2016;79(suppl 1):S3-S7.

29. Missios S, Bekelis K, Barnett GH. Renaissance of laser interstitial thermal ablation. Neurosurg Focus. 2015;38(3):E13. 
30. Pruitt R, Gamble A, Black K, et al. Complication avoidance in laser interstitial thermal therapy: lessons learned. J Neurosurg. 2017;126(4):1238-1245.

31. Ho AL, Miller KJ, Cartmell S, et al. Stereotactic laser ablation of the splenium for intractable epilepsy. Epilepsy Behav Case Rep. 2016;5:23-26.

32. Singh H, Essayed WI, Deb S, et al. Minimally invasive robotic laser corpus callosotomy: a proof of concept. Cureus. 2017;9(2):e1021.

33. Karsy M, Patel DM, Halvorson K, et al. Anterior two-thirds corpus callosotomy via stereotactic laser ablation. Neurosurg Focus. 2018;44(VideoSuppl2):V2.

\section{Disclosures}

The authors report no conflict of interest concerning the materials or methods used in this study or the findings specified in this paper.

\section{Author Contributions}

Conception and design: Dolce, Price. Acquisition of data: all authors. Analysis and interpretation of data: Caruso, Price.

Drafting the article: Caruso, Janjua. Critically revising the article:
Caruso, Dolce, Price. Reviewed submitted version of manuscript: Caruso, Dolce, Price. Statistical analysis: Caruso. Administrative/ technical/material support: Dolce, Price. Study supervision: Price.

\section{Supplemental Information}

Previous Presentations

Portions of this work were presented at the American Society of Pediatric Neurosurgery Annual Meeting in Maui, HI, January 2019, and at the American Association of Neurological Surgeons Annual Meeting in San Diego, CA, April 2019.

\section{Correspondence}

James P. Caruso: Children's Medical Center, University of Texas Southwestern, Dallas, TX. james.caruso@phhs.org. 